\title{
A REPORT BACK ON MORE THAN FOUR YEARS OF HIV/AIDS DISEASE MANAGEMENT IN SOUTHERN AFRICA
}

\author{
L D Regensberg, $M B C h B, M R C P$ \\ M S Hislop, $M S c$ \\ Aid for AIDS Pty (Ltd), Private Bag X1003, Claremont, 7735
}

\section{EARLY DAYS}

Prior to 1998 and the implementation of the Medical Schemes Act, beneficiaries of medical aid schemes who were living with HIV or AIDS were at a considerable disadvantage. Not only was risk rating permitted, but HIVseropositive individuals could be excluded from membership. As a result, fraudulent claims were common, and few individuals had access to effective antiretroviral therapy (ART). The problem was compounded by the high cost of the drugs, minimal benefits and, at the time, lack of experience in using ART on the part of many primary health care doctors.

Aid for AIDS (AfA) was based on the premise that the major cost driver in managing HIVIAIDS was hospitalisation for AIDS-related conditions and that ART, although costly, would be effective in terms of slowing progression of the disease, reducing morbidity and improving survival.' It was felt that making ART available by way of a disease management programme with careful monitoring, coupled with education of patients and meaningful clinical support of providers, would be cost-effective and benefit both the funders of health care and, more importantly, those beneficiaries who were living with HIV/AIDS.

The aims and objectives of AfA were published shortly after it was launched in $1998 .{ }^{2}$ In essence, AfA was to be a comprehensive and confidential disease management programme with a strong telephonic patient education and support component and managed access to ART and related drugs, prophylaxis and monitoring tests. Clinical support of providers was through well-trained clinical staff and a 'hands-on' Clinical Advisory Committee drawn from both the academic and the private sectors. Emphasis was placed on regular monitoring of $\mathrm{CD} 4$ counts and viral loads and collection of both clinical and claims data.

The first Clinical Guidelines, now in a substantially revised and expanded 4 th edition, were presented to a somewhat sceptical medical profession in a series of workshops around the country. The reasons for the scepticism were twofold. Firstly, AfA was recommending ART only when the CD 4 count was less than $350 / \mu$ or an AIDS-defining illness was present. This was the era of 'hit early and hit hard', and delaying therapy to the extent recommended was considered close to heresy by the majority of experts. Equally unacceptable to many was the use of dual therapy, which was known to be inferior to triple therapy at the time. However, dual therapy was all that was affordable for most medical schemes and, although sub-optimal, had clearly been shown to provide benefit for patients. Patients were urged to buy the third drug themselves in order to obtain the full benefit of ART, but few were able to afford this.

The major challenge facing the acceptance of the programme was the high cost of ART. Negotiations with representatives from pharmacy groups resulted in ART being made available at cost plus a dispensing fee of R50 per line item. Even so, the cost of the cheapest combination was still very high, and concerns were raised as to the viability of the programme and the ability of medical schemes to sustain these costs on an on-going basis. Our belief was that the drug costs would eventually come down, and that highly active antiretroviral therapy (HAART) would become the standard of care. Fortunately, this has proved to be the case (Table I). However, only some manufacturers have reduced ART prices substantially and only a limited range of HAART can be made available.

\section{CONSOLIDATION AND GROWTH}

The subsequent 4 years has seen a phenomenal growth in AfA (Fig. 1), from a handful of contracted medical schemes to 38 , from dealing with South African medical schemes only to providing the programme to multinational companies throughout Africa, and from offering only a disease management programme to offering a comprehensive risk management solution for corporates. Providers outside the country are able to register patients by Internet and interact with clinical staff electronically. 


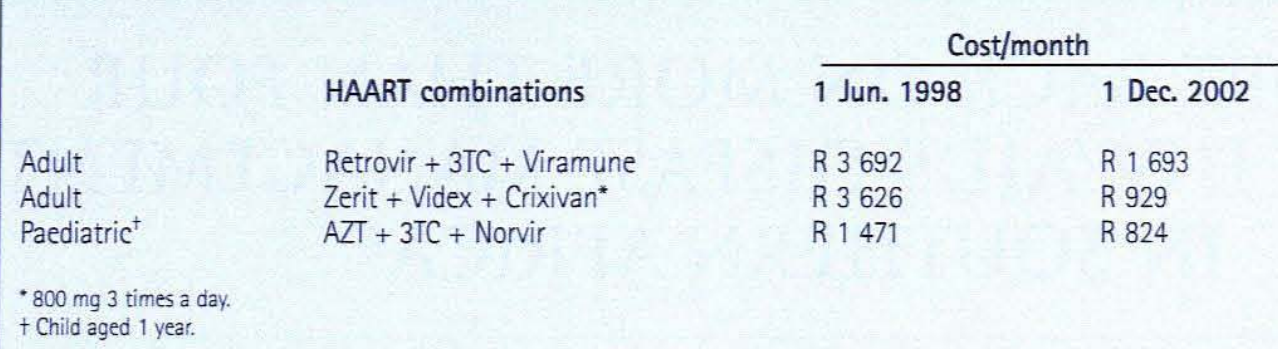

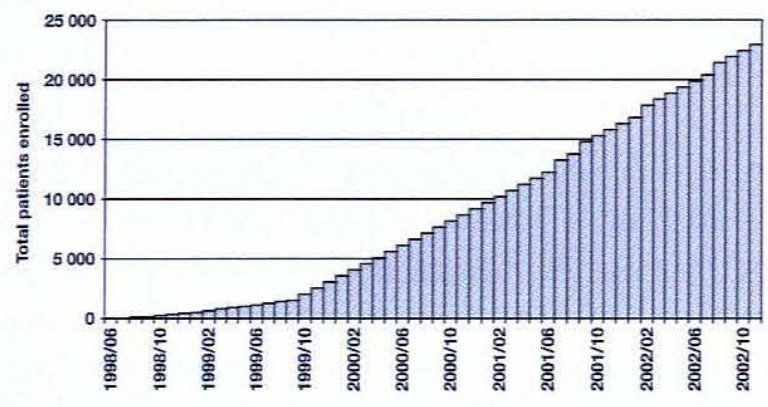

Fig. 1. Cumulative enrolment of patients onto the Aid for AIDS programme.

Well over 20000 people have been registered on AfA to date, and some 12000 are on ongoing ART (mostly HAART). Cover is provided to over 2 million beneficiaries. The staff complement has grown from 6 to 36 people, and now includes a dedicated management team, 2 full-time doctors and 12 nurses and pharmacists, as well as IT and administrative staff.

Some interesting trends have emerged from the enrolment data. Most striking is the patient gender distribution, with $61 \%$ females and $39 \%$ males. This is higher than expected from AIDS demographic modelling (the prevalence for the country as a whole is estimated to be marginally higher in adult women than men), ${ }_{1}{ }^{3}$ and may be due in part to increased awareness of HIV status in pregnant women through voluntary counselling and testing (VCT). To date, some $20 \%$ of female patients have been pregnant at registration. Another factor behind male under-enrolment may be a greater level of denial in males, as this group remains under-represented even if pregnancy is taken into account.

The age distribution of patients shows that enrolment is greatest between 25 and 45 years, with $74 \%$ of patients in this age group (Fig. 2). In total, 93\% of patients are older than 19. There is a surprisingly large difference between enrolment in 15 - 19-year-olds and in 20 - 24-year-olds $(0.14 \%$ v. $0.70 \%)$, as well as between the latter and $25-29$ year-olds $(0.70 \%$ v. $2.07 \%)$. This may be because the majority of the younger patients are dependants - 100\% and $85 \%$ in 15 - 19- and 20 - 24-year-olds respectively, compared with $30 \%$ in 25 - 29-year-olds. There may therefore be a reluctance in these age groups to come

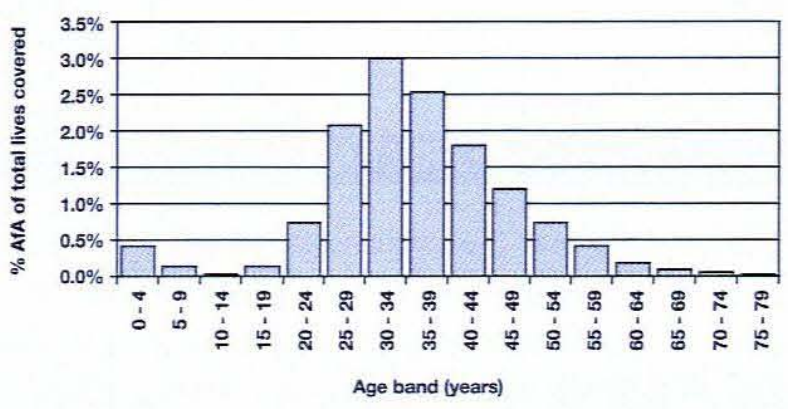

Fig. 2. Aid for AIDS enrolment by age ( $\%$ total lives covered).

forward out of fear of disclosing status to the main member.

Enrolment by region shows that the rate of uptake is highest in KwaZulu/Natal (1.55\%), and lowest in the Western Cape $(0.15 \%)$, with more than a tenfold difference between these regions (Fig. 3). The rate of enrolment per region will be influenced by the underlying HIV prevalence in the medical scheme membership in that region, as well as localised factors, such as the level of stigmatisation, which determine the probability of disclosure.

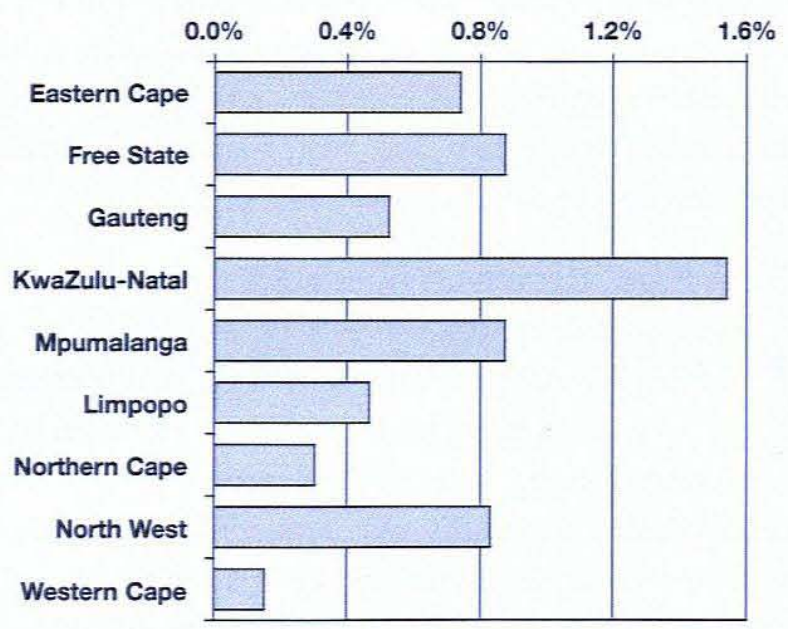

Fig. 3. Aid for AIDS enrolment by region ( $\%$ total lives covered).

\section{MTCT OUTCOMES}

AfA currently has polymerase chain reaction (PCR) results for 962 infants where complete mother-to-child transmission (MTCT) prophylaxis was provided, mostly 
short-course zidovudine for the mother, 6 weeks of zidovudine for the child, and milk substitutes for 6 months (Table II). Most babies were delivered by caesarean section. The transmission rate of 5.3\% is similar to the rate reported in an earlier study. ${ }^{*}$ Over the past year the use of shortcourse HAART has been recommended, and the reduction in MTCT is expected to improve further.

\begin{tabular}{|lcc|}
\hline \multicolumn{3}{c|}{ TABLE II. MTCT PCR RESULTS } \\
\hline PCR & Total & $\%$ \\
Negative & 911 & 94.7 \\
Positive & 51 & 5.3 \\
\hline
\end{tabular}

\section{VIROLOGICAL RESPONSE}

The virological response of AfA patients to antiretroviral therapy is shown in Fig. 4. Most patients started on dual therapy then switched to HAART. The mean baseline viral load of patients at commencement of antiretroviral therapy was $5.01 \log _{10}$. It is interesting to note the improved response to therapy from 27 months onwards. This is attributed to a greater proportion of patients with data at these time points having had therapy intensified to HAART. The virological response at one year $\left(1 \log _{10}\right.$ reduction) is associated with a $90 \%$ reduction in disease progression in patients on dual therapy. ${ }^{5}$

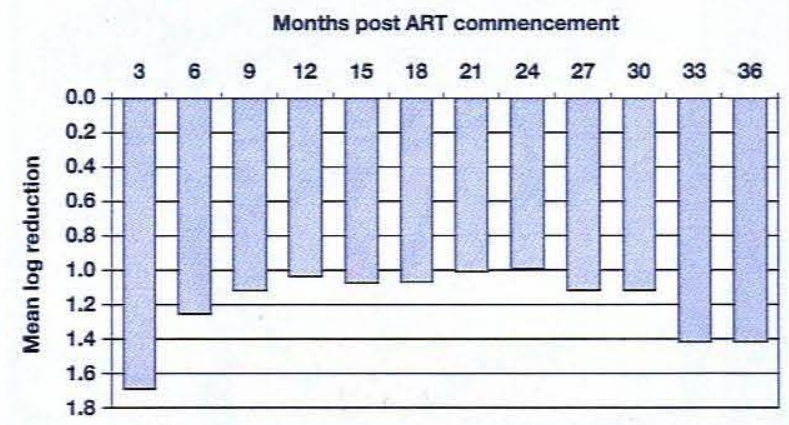

Fig. 4. Virological response to antiretroviral therapy in Aid for AIDS patients.

\section{CD4 COUNT AT REGISTRATION}

The $\mathrm{CD} 4$ count at registration provides a useful measure of whether patients have enrolled late (Table III). Ideally, patients should register well before their CD4 count reaches 200 cells/ $\mu$ l so that ART can be commenced at the optimal time. It is of concern that a large percentage of patients enter the programme with a CD4 count $<200$ cells/ $/ \mu$. This is either because patients are ignorant of their HIV status until they are admitted to hospital for an opportunistic infection, or because they are in denial. It is interesting that on average, women lexcluding those who are pregnant at registration) tend to enrol at an earlier CD4 stage than their male counterparts. To date, $43 \%$ of non-
TABLE III. CD4 COUNT DISTRIBUTION AT ENROLMENT ON THE AID FOR AIDS PROGRAMME

$\begin{array}{ll}\text { CD4 (cells/ } \mu l) & \% \\ >499 & 15 \\ 350-499 & 15 \\ 200-349 & 26 \\ 50-199 & 29 \\ <50 & 15\end{array}$

pregnant female patients have enrolled with a CD4 count $<200$ cells $/ \mu \mid$, in comparison with $50 \%$ of males. Preliminary results point to entry CD4 count as being a strong determinant of survival. In particular, patients with a CD4 count $<50$ cells/ $\mu$ l appear to have a substantially higher probability of death within 24 months of enrolment.

\section{HOSPITALISATION}

The hospitalisation data illustrate that admission is frequently the event which precipitates AfA registration (Fig. 5). Were all patients to commence ART at the optimal time, we would not expect to see a peak in admissions around the enrolment period. The programme has been monitoring admission rates after commencement of ART for over 4 years. It has been heartening to see that once patients have commenced therapy, the admission rate has stabilised. This is particularly notable considering that the majority of patients enrolled at a relatively late stage of the disease. Effectively this has translated into a stable postregistration cost scenario rather than a steady upward trend in subsequent years.

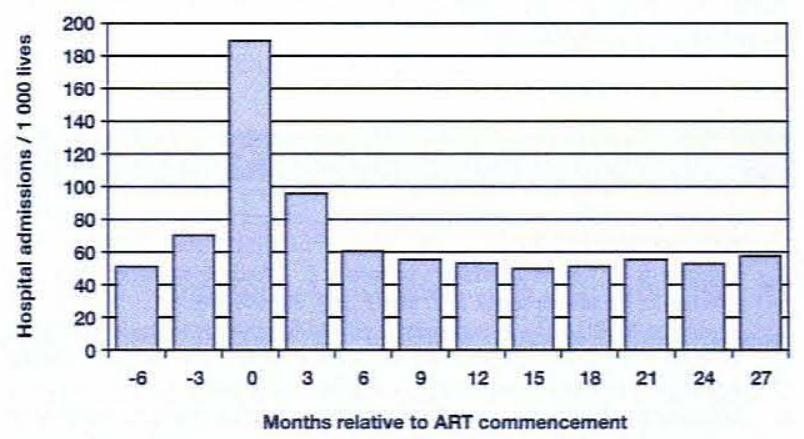

Fig. 5. Hospital admissions relative to commencement of ART.

\section{THE FUTURE}

The aim of any disease management programme (DMP) is evidence-based case management with continual evaluation of clinical and economic outcomes, in order to optimise the use of increasingly scarce health care resources, limit disease progression and improve overall health.

Our outcomes show that access to ART reduces the need for hospitalisation and provides significant reduction in viral load over 3 years despite starting with sub-optimal 
therapy. We aim to assess the impact of cost reductions in increasing access to HAART on survival and hope to present these data in due course. The programme also offers a valuable education and awareness opportunity for beneficiaries and employees of contracted medical schemes and companies, as well as clinical support for those doctors who require it.

We have identified a number of challenges. These include communicating more effectively with patients, maintaining adherence to therapy, and, in common with other HIV $\mathrm{DMP}^{6}$ addressing the relatively low level of enrolment of earlier stage patients. We would like to reverse the current situation where some patients only join the programme after they are hospitalised for an AIDS-related condition.

It is crucial for all health care workers, including doctors, to encourage as many people as possible to know their status and if positive, to join their scheme or company's HIV DMP long before they are likely to develop an opportunistic condition.

Continuing to provide optimal therapy within the available benefit remains an on-going problem as medical schemes come under increasing pressure from spiralling health care inflation. This will become even more critical as patients move on to more complex salvage regimens after a number of years and the need for sophisticated investigations such as resistance testing increases.

After more than 4 years AfA has demonstrated that it is possible to manage HIV costs effectively, and despite the challenges we have identified, there is no reason why HIV disease management cannot be extended to far larger numbers of people.

\section{REFERENCES}

Palella FJ، Delaney KM, Moorman AC, et al. Declining morbidity and mortality among patients with advanced human immunodeficiency virus infection. $N$ Eng/J Med 1998; 338: 853-860.

2. Regensberg $L D$, Cowlin $J R$, Walters $L$, Ramsay $G$. Affordable management of HIV infection in the private sector. SAfr Med J 1998; 88: 945-948.

3. Dorrington RE, Bradshaw D, Budlender D. HIV/AIDS Profile of the Provinces of South Africa - Indicators for 2002. Cape Town: Centre for Actuarial Research, Medical Research Council and Actuarial Society of South Africa, 2002.

4. Regensberg L, Pead C, Mahumapelo I, Cotton M. Prevention of vertical transmission of human immunodeficiency type 1 virus in a managed care setting. S Afr Med $\perp$ 2000; 90: 1092-1093.

Katzenstein DA, Hammer SM, Hughes MD, et al. The relation of virologic and immunologic markers to clinical outcomes after nucleoside therapy in HIV-infected adults with 200 to $500 \mathrm{CD} 4$ cells per cubic millimeter. AIDS Clinical Trials Group Study 175 Virology Study Team. N Engl J Med 1996; 335: 1091-1098.

6. Stein A, McLeod H, Achmat Z. The cover provided for HIV/AIDS benefits in medical schemes in 2002. UCT-CARE/TAC Industry Survey: CARE Monograph 10, July 2002.

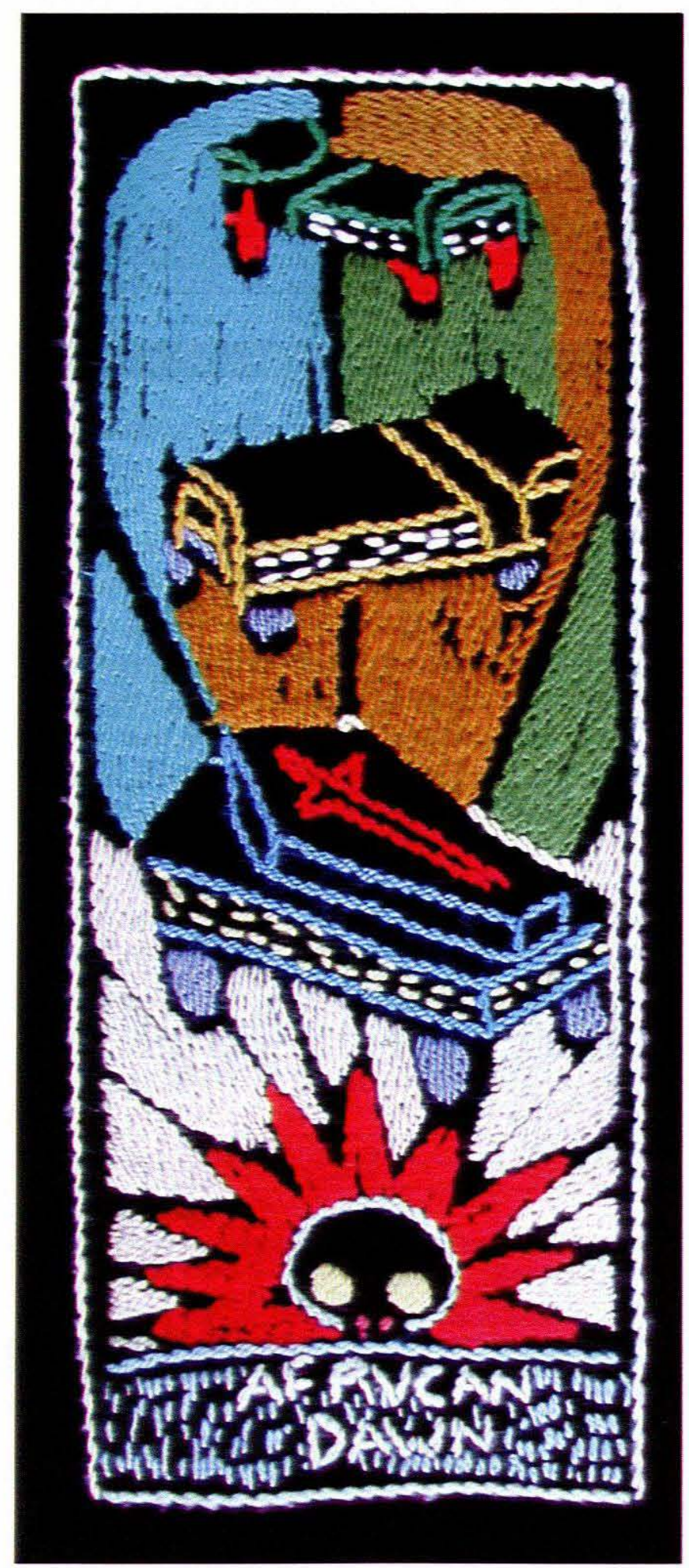

